\title{
Review
}

\section{Molecularly-Targeted Therapies in Gynecologic Cancer}

\author{
Kathryn Kennedy, MD; William Robinson, MD* \\ Tulane Cancer Center, Tulane University School of Medicine, New Orleans, LA, USA
}

\section{${ }^{*}$ Corresponding author}

William Robinson, MD

Professor, Tulane University School of Medicine, SL-II 1430 Tulane Avenue, New Orleans, LA 70I I5, USA; E-mail: wrobinso@tulane.edu

\section{Article information}

Received: December 19

\section{Cite this article}

Kennedy K, Robinson W. Molecularly-targeted therapies in gynecologic cancer. Gynecol Obstet Res Open J. 20I8; 5(I): I-5. doi: I0.17/40/GOROJ-5-I45

\section{ABSTRACT}

The development of chemotherapy agents that precisely target specific molecular structures in cancer cells have become a priority in oncology research. In principle, this method halts cancer cell proliferation while allowing normal function of healthy cells. These molecular-based chemotherapy agents target many types of molecules involved in the growth, spread, and survival of malignant cells. Several of these target molecules have been identified in female genital tract malignancies, and multiple agents targeted at those molecules have been developed as a treatment. This review outlines three major types of targeted agents that have clinical relevance in the treatment of gynecologic cancer. The first group of drugs inhibits vascular endothelial growth factor (VEGF), which normally facilitates angiogenesis. The second group inhibits poly (ADP-ribose) polymerase (PARP), a base-excision enzyme that repairs single-strand DNA breaks. The final category is a set of drugs that inhibit programmed-cell death protein 1 , an immune checkpoint that normally prevents autoimmunity. Therapeutic benefit has been demonstrated for each of these drug types in gynecologic, and particularly ovarian, cancers. New agents, and applications for these agents have been developing at a rapid pace in each of these categories. Food and Drug Administration (FDA) approval has been accelerated for several of these agents in recent years, suggesting a significant change in the process by which new drugs enter the clinical armamentarium. In short, the development of molecularly-targeted drugs for the treatment of cancer is a promising and rapidly-moving field.

\section{Keywords}

Chemotherapy; Poly (ADP-ribose) polymerase (PARP); Gynecologic.

\section{Abbreviations}

VEGF: Vascular Endothelial Growth Factor; FDA: Food and Drug Administration; PARP: Poly (ADP-ribose) Polymerase; GOG: Gynecologic Oncology Group; ORR: Objective Response Rates; PFS: Progression-Free Survival.
\end{abstract}

\section{INTRODUCTION}

$\mathrm{H}$ istorically, chemotherapy for cancer have been based on the use of cytotoxic agents that kill rapidly-dividing cells, typically including at least some non-malignant cells. The advent of agents targeted at specific molecules associated with cancer has been revolutionary, and have generally demonstrated much less toxicity than traditional chemotherapy, with varying (but often significant) degrees of effectiveness. ${ }^{1,2}$ Targets include molecules for signal transduction, gene expression, apoptosis inhibition, angiogenesis inhibition, and immunomodulation. Since cancer cells rely on these molecules for proliferation and survival targets that precisely inhibit those molecules could stop malignant progression.
These principles also apply to gynecologic malignancies, and there is a great need for novel therapeutic agents in these cancers. This is especially true for ovarian cancer. Nearly $80 \%$ of women diagnosed with epithelial ovarian cancer, for example, will achieve a complete response to traditional cytotoxic chemotherapy (generally a combination of cis- or carboplatin and a taxane). However, in the majority of cases, these responses are not durable, and as many as $75 \%$ will eventually succumb to the disease, demonstrating the urgent need for new treatments. Thus far, three molecular targets have demonstrated efficacy in gynecologic malignancies. Vascular endothelial growth factor (VEGF), which normally facilitates angiogenesis, is the target of bevacizumab, an agent that is FDA-approved for cervical, ovarian, fallopian tube, 
and primary peritoneal malignancy. Poly (ADP-ribose) polymerase (PARP), which normally repairs single-strand DNA breaks, is the target for niraparib, olaparib, and rucaparib, which are Food and Drug Administration (FDA)-approved for ovarian, fallopian tube, and primary peritoneal cancer. ${ }^{3}$ Finally, PD-1, the cell surface protein which is responsible for programmed cell death, has been targeted by pembrolizumab and has been FDA-approved for the treatment of multiple solid malignancies, including ovarian and cervical cancer. Avelumab is another anti-PD-1 agent for which two phase III clinical trials are currently in progress. It is important to note; however, that these drugs are not without significant toxicities, however, despite their targeted design. The toxicities of each are generally related to the blockade of the molecular target in question. For VEGF inhibitors, toxicities include hypertension, bleeding, and even bowel perforation. ${ }^{4}$ For PARP inhibitors, GI upset as well as hematologic toxicities such as anemia, thrombocytopenia, myelodysplastic syndrome, and acute myeloid leukemia are possible. ${ }^{5}$ PD-1 inhibitor toxicities are generally common but mild, and include rash and pruritis, arthralgia and myalgia, as well as potential thyroid toxicities. ${ }^{6}$ The discussion that follows reviews each of these of molecular chemotherapies and their roles in gynecologic cancer.

\section{CONCLUSIONS}

\section{VEGF Inhibitors}

The first category of molecular chemotherapies is VEGF inhibitors, of which bevacizumab is the predominant drug and first of its kind to become commercially available in the United States. Cancer cells rely on VEGF to stimulate angiogenesis through endothelial cell stimulation in settings of oxygen deprivation. Bevacizumab is a recombinant humanized monoclonal antibody that binds VEGF in the bloodstream, therefore preventing it from binding to its cell surface receptor and stimulating angiogenesis. ${ }^{7}$ In 2014, bevacizumab was approved by the FDA for intravenous (IV) infusion in combination with paclitaxel, liposomal doxorubicin, or topotecan for the treatment of platinum-resistant epithelial ovarian, fallopian tube, and primary peritoneal cancer. This was based on the AURELIA study, an international, two-arm trial in which subjects were randomized to receive one of the above-mentioned drugs with or without bevacizumab. This trial showed a statistically significant progression-free survival (PFS) of 6.8 months for the subjects who received bevacizumab, compared with 3.4 months for those who did not. ${ }^{8}$ Bevacizumab was also approved for platinum-sensitive epithelial ovarian, fallopian tube, and primary peritoneal cancers based on the GOG 213 and OCEANS studies. In Gynecologic Oncology Group (GOG) study \#213, bevacizumab plus chemotherapy (carboplatin and paclitaxel) demonstrated an increase in median overall survival compared with chemotherapy alone (42.6 vs. 37.3 months). In the OCEANS study, bevacizumab plus chemotherapy (carboplatin and gemcitabine) resulted in significant improvement in PFS compared with chemotherapy alone (12.4 vs. 8.4 months). ${ }^{9}$ Also in 2014, bevacizumab was approved for the treatment of persistent, recurrent, or metastatic cervical cancer in combination with paclitaxel, cisplatin, or topotecan, based on GOG 240, which compared PFS of bevacizumab with chemotherapy to chemotherapy alone. This study demonstrated a statistically significant PFS (16.8 vs. 12.9 months) in the group receiving bevacizumab. ${ }^{10}$ Since then, bevacizumab, has become a mainstay of both ovarian and cervical cancer treatment. A second VEGF inhibitor, cediranib, has also shown promise; results from a phase II clinical trial show that the combination of cediranib with the PARP inhibitor olaparib is significantly more effective against recurrent platinum-sensitive ovarian cancer with BRCA mutations than olaparib alone (PFS of 17.7 vs. 9 months). ${ }^{11}$ VEGF inhibitors are not without side effects; however, including hypertension, fatigue, bleeding (particularly nosebleeds), headache, rash, bowel perforation and fistula formation in up to $3 \%$ of patients. $^{4}$

\section{PARP Inhibitors}

The second major category of molecular chemotherapy agents for gynecologic cancers is the PARP inhibitors. PARPs are enzymes that perform base excisions in damaged DNA, thus repairing single-strand breaks. Inhibiting PARP allows these breaks to accumulate, which in turn leads to double-stranded breaks when the DNA replicates. ${ }^{12}$ In normal cells, these breaks can still be repaired by homologous recombination. Tumor suppressor proteins BRCA1 and 2 play a substantial role in this process, and thus cancer cells with defective BRCA1 and 2 are unable to perform this repair. ${ }^{13}$ The accumulation of double-stranded breaks then leads to cell death. ${ }^{14,15}$ A second mechanism by which PARP inhibitors work has also been described and has been termed a 'poisoning' mechanism. When the PARP inhibitor and PARP are bound, PARP is unable to dissociate from DNA. The inhibitor-PARP-DNA complexes then accumulate, which causes cytotoxicity. For this reason, the use of PARP inhibitors is not reliant on the first, catalytic mechanism or limited to BRCA1 and 2-mutated cancers. ${ }^{16}$ Three oral PARP inhibitors have been approved by the FDA for use in ovarian cancer: olaparib in December 2014, rucaparib in December 2016, and niraparib in March 2017. Each of these agents varies in their efficacy as a catalytic PARP inhibitor and as a PARP 'poison,' as shown in Table 1. Each agent's ability to inhibit the catalytic activity of PARP is independent of its potency as a PARP 'poison'. Olaparib, for example, has been consistently shown to be a potent catalytic PARP inhibitor, but is not as potent a cytotoxic agent as niraparib. For this reason, they have different clinical indications, as shown in Table 1. Also shown in Table 1 are the studies upon which FDA approval was based. Of note, the more recent agents have received special designations for accelerated approval, and are no longer necessarily subject to the typical FDA requirement of having completed two Phase III clinical trials. ${ }^{17}$ Side effects of these agents include nausea, vomiting, fatigue, anemia, thrombocytopenia, myelodysplastic syndrome, and acute myeloid leukemia. Several additional PARP inhibitors are increasingly clinically relevant, such as veliparib (ABT-888), which was associated with a median PFS of 8.18 months in a phase II trial examining treatment of BRCA1/2 mutated ovarian, fallopian tube, and primary peritoneal cancers. Phase III trials are currently in progress. ${ }^{5}$

\section{PD-I Inhibitors}

The final class of molecular chemotherapy agents discussed in this review is the PD-1 inhibitor group. PD-1 is a cell surface receptor expressed on T-cells. Normally, PD-1 binds to ligands PD-L1 and 


\begin{tabular}{|c|c|c|c|}
\hline & Niraparib (MK-4827) & Olaparib & Rucaparib \\
\hline Potency to inhibit catalytic PARP activity & Weak & Strong & Not studied \\
\hline Potency as a cytotoxic PARP poison & Strong & Intermediate & Not studied \\
\hline FDA Approved for: & $\begin{array}{l}\text { Maintenance therapy for platinum-sensitive } \\
\text { recurrent high-grade serous epithelial } \\
\text { ovarian, fallopian tube, primary peritoneal } \\
\text { cancer with or without BRCA mutations } \\
\text { (March 2017) }\end{array}$ & $\begin{array}{l}\text { Monotherapy for germline BRCA- } \\
\text { mutated ovarian cancer that has failed } 3 \\
\text { or more previous lines of chemotherapy } \\
\text { (December 20I4) } \\
\text { Maintenance therapy for platinum- } \\
\text { sensitive recurrent epithelial ovarian, fal- } \\
\text { lopian tube, or primary peritoneal cancer } \\
\text { (August 20I7) }\end{array}$ & $\begin{array}{l}\text { Monotherapy for germline, somatic, or } \\
\text { noninherited BRCA-mutated ovarian } \\
\text { cancer that has failed } 2 \text { or more previ- } \\
\text { ous lines of chemotherapy (December } \\
\text { 2016) }\end{array}$ \\
\hline Trials upon which the FDA based approval & $\begin{array}{l}\text { NOVA Trial (Phase III): } \\
\text { PFS in the gBRCA mut cohort was signifi- } \\
\text { cantly increased with niraparib ( } 21.0 \text { vs } 5.5 \\
\text { months) and PFS in the non-gBRCAmut } \\
\text { cohort was also increased ( } 9.3 \text { vs. } 3.9 \\
\text { mo). }{ }^{18}\end{array}$ & $\begin{array}{l}\text { Monotherapy: } \\
\text { Kaufman, et al (Phase II) } \\
\text { Maintenance: } \\
\text { SOLO-2 (Phase III) showed PFS I9.I vs. } \\
5.5 \mathrm{mo}^{19} \\
\text { Study } 19 \text { (Phase III) showed PFS of } 8.4 \\
\text { vs. } 4.8 \mathrm{mo}^{20}\end{array}$ & $\begin{array}{l}\text { Study } 10 \text { and and Ariel } 2 \text { (Phase II, in } \\
\text { progress and so their results were com- } \\
\text { piled) Assessed thus far is the Objective } \\
\text { Response Rate, } 54 \% \text { in } 106 \text { patients }{ }^{21}\end{array}$ \\
\hline Special FDA Designations & $\begin{array}{l}\text { Fast Track, Priority Review, Breakthrough } \\
\text { Therapy, Orphan Drug }\end{array}$ & $\begin{array}{l}\text { Accelerated Approval for monotherapy } \\
\text { Priority Review for maintenance therapy }\end{array}$ & $\begin{array}{l}\text { Breakthrough Therapy, Priority Review, } \\
\text { and Orphan drug }\end{array}$ \\
\hline
\end{tabular}

PD-L2, which reduces T-cell proliferation, T-cell signaling, cytokine production, and increases T-cell susceptibility to apoptosis. This prevents pathologic immune response in healthy patients and thus prevents autoimmunity. When malignant cells express these ligands; however, they are allowed to proliferate uninhibited by these T-cell mechanisms. PD-1 inhibitors shut down this checkpoint by preventing the binding of PD-1 to its ligands, thus allowing effector T-cells to mount an immune response against the tumor. ${ }^{22}$ The FDA has recently approved Pembrolizumab for use in multiple solid tumors, including gynecologic cancers, which express this marker. Plus, several agents of this type are currently being evaluated in phase III clinical trials. Because initial studies showed that ovarian cancers with high expression of PD-L1 were associated with poorer outcomes, PD-1 inhibitors have been selected as a potential molecular target for ovarian cancer treatment. ${ }^{23}$ Nivolumab and Pembrolizumab are anti-PD-1 IgG4 monoclonal antibodies that have shown $15 \%$ and $11.5 \%$ objective response rates (ORR) in phase II and phase Ib studies of ovarian cancer patients, respectively. ${ }^{24,25}$ Avelumab is an anti-PDL1 IgG1 monoclonal antibody that showed an ORR of $9.7 \%$ in patients with refractory or recurrent ovarian cancer in a phase Ib study. ${ }^{26}$ This has led to two-phase III studies; the first is avelumab for front-line therapy in ovarian cancer in combination with carboplatin and paclitaxel (Javelin ovarian 100), and the second is avelumab for recurrent platinumresistant disease (Javelin ovarian 200). ${ }^{27}$ Other anti-PDL1 agents in earlier stages of development for ovarian cancer treatment include the IgG1 monoclonal antibodies durvalumab and atezolizumab. ${ }^{28}$ While side effects for this genre of drugs are common (about $40 \%$ of patients) they are generally not severe (only 5\% using Common Terminology Criteria for Adverse Events), and include rash, pruritis, diarrhea, arthralgia, arthritis, myalgia, myositis, thyroiditis, hypothyroidism, hypophysitis, and infusion-related reactions. ${ }^{6,29}$

As the above discussion shows there are numerous promising possibilities for molecular chemotherapies in the treatment of gynecologic malignancies, and particularly in ovarian cancer.
VEGF inhibitors, PARP inhibitors, and PD-1 inhibitors are the leading molecular chemotherapy agents of this type. The development of these agents has made rapid progress in the past decade, in part due to the accelerated-approval designations the FDA has assigned to many of them. Rucaparib, for instance, received Breakthrough Therapy, Priority Review, and Orphan Drug designations, and was approved without completion of any phase III trials. New applications for existing drugs are also in rapid development, such as the combination of olaparib with radiotherapy, which has already shown that low doses of olaparib cause radiosensitization and therefore may be a new and valuable type of treatment for ovarian cancer. ${ }^{30}$

In conclusion, acceleration of the approval process for these agents has not been taken lightly by the FDA. Unexpected outcomes or toxicities have occurred in the wake of expedited approvals. However, the high mortality of ovarian cancer, plus the fact that phase III trials often require years to complete, suggest that changes in the approval process would be very well-received by those affected by this disease. Furthermore, the fast pace at which new drugs are being developed means that by the time phase III trials are completed, newer, possibly superior, drugs may exist. The use of surrogate molecular markers as endpoints in the place of traditional measures of progression-free and overall survival is being carefully evaluated. Patient-reported outcomes are also becoming much more important to the process of drug development and approval. ${ }^{31}$ If drug development continues at this rate, an overall restructuring of the drug approval process may become necessary and reasonable. In conclusion, the development of molecular-based chemotherapies for gynecologic cancers is a rapidly progressing field and many of these drugs have proven thus far to be very promising in the treatment of these devastating illnesses.

\section{CONFLICTS OF INTEREST}

The authors declare that they have no conflicts of interest. 


\section{REFERENCES}

1. Tsuda N, Watari H, Ushijima K. Chemotherapy and molecular targeting therapy for recurrent cervical cancer. Chin J Cancer Res. 2016; 28(2): 241-253. doi: 10.21147/j.issn.1000-9604.2016.02.14

2. Rojas V, Hirshfield K, Ganesan S, Rodriguez-Rodriguez L. Molecular characterization of epithelial ovarian cancer: Implications for diagnosis and treatment. Int J Mol Sci. 2016; 17(12): 2113. doi: $10.3390 /$ ijms 17122113

3. Murai J, Huang S, Brata Das B, et al. Differential trapping of PARP1 and PARP2 by clinical PARP inhibitors. Cancer Res. 2012; 72(21): 5588-5599. doi: 10.1158/0008-5472.CAN-12-2753

4. Sliesoriaitis S, Tawfik B. Bevacizumab-induced bowel perforation. J Am Osteopath Assoc. 2011; 111(7): 437-444.

5. Coleman RL, Sill MW, Bell-McGuinn K, et al. A phase II evaluation of the potent, highly selective PARP inhibitor veliparib in the treatment of persistent or recurrent epithelial ovarian, fallopian tube, or primary peritoneal cancer in patients who carry a germline BRCA1 or BRCA2 mutation-an NRG oncology/gynecologic oncology group study. Gynecol Oncol. 2015; 137(3): 386-391. doi: 10.1016/j.ygyno.2015.03.042

6. Brahmer JR, Tykodi SS, Chow LQ, et al. Safety and activity of anti-PD- L1 antibody in patients with advanced cancer. $N$ Engl J Med. 2012; 366(26): 2455-2465. doi: 10.1056/NEJMoa1200694

7. Los M, Roodhart JML, Voest E. Target practice: Lessons from phase III trials with bevacizumab and vatalanib in the treatment of advanced colorectal cancer. Oncologist. 2007; 12(4): 443-450. doi: $10.1634 /$ theoncologist.12-4-443

8. Pujade-Lauraine E, Hilpert F, Weber B, et al. Bevacizumab combined with chemotherapy for platinum-resistant recurrent ovarian cancer: The AURELIA open-label randomized phase III trial. $J$ Clin Oncol. 2014; 32(35): 4025. doi: 10.1200/JCO.2013.51.4489

9. Aghajanian C, Blank SV, Goff BA, et al. OCEANS: a randomized, double-blind, placebo-controlled phase III trial of chemotherapy with or without bevacizumab in patients with platinumsensitive recurrent epithelial ovarian, primary peritoneal, or fallopian tube cancer. J Clin Oncol. 2012; 30(17): 2039-2045. doi: 10.1200/JCO.2012.42.0505

10. Tewari KS, Sill MW, Long HJ $3^{\text {rd }}$, et al. Improved survival with bevacizumab in advanced cervical cancer. N Engl J Med. 2014; 370(8): 734-743. doi: 10.1056/NEJMoa1309748

11. Liu JF, Barry WT, Birrer M, et al. Combination cediranib and olaparib versus olaparib alone for women with recurrent platinumsensitive ovarian cancer: A randomised phase 2 study. Lancet Oncol. 2014; 15(11): 1207-1214. doi: 10.1016/S1470-2045(14)70391-2

12. Dantzer F, de La Rubia G, Menissier-De Murcia J, et al. Base excision repair is impaired in mammalian cells lacking poly (ADP- ribose) polymerase-1. Biochemistry. 2000; 39: 7559-7569. doi: 10.1021/bi0003442

13. Gudmundsdottir K, Ashworth A. The roles of BRCA1 and BRCA2 and associated proteins in the maintenance of genomic stability. Oncogene. 2006; 25: 5864-5874. doi: 10.1038/sj.onc.1209874

14. Fong P, Boss D, Yap TA, et al. Inhibition of poly (ADP-ribose) polymerase in tumors from brca mutation carriers. N Engl J Med. 2009; 361: 123-134. doi: 10.1056/NEJMoa0900212

15. Ashworth A. A synthetic lethal therapeutic approach: Poly (ADP ribose) polymerase inhibitors for the treatment of cancers deficient in DNA double-strand break repair. J Clin Oncology. 2008; 26: 3785-3790. doi: 10.1200/JCO.2008.16.0812

16. Murai J, Huang S, Das BB, et al. Differential trapping of PARP1 and PARP2 by clinical PARP inhibitors. Cancer Research. 2012; 72(21): 5588-5599. doi: 10.1158/0008-5472.CAN-12-2753

17. Murai J, Huang S, Das BB, et al. Differential trapping of PARP1 and PARP2 by clinical PARP inhibitors. Cancer Research. 2012; 72(21): 5588-5599. doi: 10.1158/0008-5472.CAN-12-2753

18. Mirza MR, Monk BJ, Herrstedt J, et al. ENGOT-OV16/NOVA Investigators. Niraparib maintenance therapy in platinum-sensitive, recurrent ovarian cancer. N Engl J Med. 2016; 375(22): 2154-2164. doi: 10.1056/NEJMoa1611310

19. Pujade-Lauraine E, Ledermann JA, Penson RT, et al. Treatment with olaparib monotherapy in the maintenance setting significantly improves progression-free survival in patients with platinum-sensitive relapsed ovarian cancer: Results from the phase III SOLO2 study. Society of Gynecologic Oncologists Annual Meeting. 2017.

20. Ledermann J, Harter P, Gourley C, et al. Olaparib maintenance therapy in platinum-sensitive relapsed ovarian cancer. $N$ Engl $J$ Med. 2012; 366: 1382-1392. doi: 10.1056/NEJMoa1105535

21. Shapira-Frommer R, Oza A, Domchek SM, et al. 2746 A Phase 2 open-label, multicenter study of single-agent rucaparib in the treatment of patients with relapsed ovarian cancer and a deleterious BRCA mutation. Eur J Cancer. 2015; 51: S545. doi: 10.1016/ S0959-8049(16)31512-X

22. Francisco LM, Sage PT, Sharpe AH. The PD-1 pathway in tolerance and autoimmunity. Immunological Reviews. 2010; 236: 219-242. doi: 10.1111/j.1600-065X.2010.00923.x

23. Hamanishi J, Mandai M, Iwasaki M, et al. Programmed cell death 1 ligand 1 and tumor-infiltrating CD8+ T lymphocytes are prognostic factors of human ovarian cancer. Proc Natl Acad Sci USA. 2007; 104(9): 3360-3365. doi: 10.1073/pnas.0611533104

24. Hamanishi J, Mandai M, Ikeda T, et al. Safety and antitumor activity of anti-PD-1 antibody, nivolumab, in patients with platinumresistant ovarian cancer. J Clin Oncol. 2015; 33(34): 4015-4022. doi: doi: 10.1200/JCO.2015.62.3397 
25. Varga A, Piha-Paul SA, Ott PA, et al. Antitumor activity and safety of pembrolizumab in patients (pts) with PD-L1 positive advanced ovarian cancer: Interim results from a phase Ib study. J Clin Oncol. 2015; 33(suppl; abstr 5510). doi: 10.1200/jco.2015.33.15 suppl.5510

26. Disis ML, Patel MR, Pant S, et al. Avelumab (MSB0010718C; anti-PD- L1) in patients with recurrent/refractory ovarian cancer from the JAVELIN Solid Tumor phase Ib trial: Safety and clinical activity. J Clin Oncol. 2016; 34 (suppl; abstr5533). doi: 10.1200/ JCO.2016.34.15_suppl.5533

27. Gaillard S, Secord AA, Monk B. The role of immune checkpoint inhibition in the treatment of ovarian cancer. Gynecologic Oncology Research and Practice. 2016; 3: 11. doi: 10.1186/s40661-0160033-6

28. Lee J, Zimmer AD, Lipkowitz S, et al. Phase I study of the PDL1 inhibitor, durvalumab (MEDI4736; D) in combination with a
PARP inhibitor, olaparib (O) or a VEGFR inhibitor, cediranib (C) in women's cancers (NCT02484404). J Clin Oncol. 2016; 3(suppl; abstr 3015). doi: 10.1200/JCO.2016.34.15_suppl.3015

29. Topalian SL, Hodi FS, Brahmer JR, et al. Safety, activity, and immune correlates of anti-PD- 1 antibody in cancer. $N$ Engl J Med. 2012; 366(26): 2443-2454. doi: 10.1056/NEJMoa1200690

30. Verhagen CVM, de Haan R, Hageman F, et al. Extent of radiosensitization by the PARP inhibitor olaparib depends on its dose, the radiation dose and the integrity of the homologous recombination pathway of tumor cells. Radiother Oncol. 2015; 116(3): 358-365. doi: 10.1016/j.radonc.2015.03.028

31. Wyndham H, Wilson DP, Schenkein CL, Jernigan JW, Schilsky RL. Reevaluating the accelerated approval process for oncology drugs. Clin Cancer Res. 2013; 19(11): 2804-2809. doi: 10.1158/10780432.CCR-13-0315 